\title{
MicroRNA-592 promotes cell proliferation, migration and invasion in colorectal cancer by directly targeting SPARC
}

\author{
ZHENGUO PAN, RUI XIE, WEI SONG and CHENGCHENG GAO \\ Department of Gastroenterology, The Affiliated Huai'an No. 1 People's Hospital of \\ Nanjing Medical University, Huai'an, Jiangsu 223300, P.R. China
}

Received May 1, 2020; Accepted December 8, 2020

DOI: $10.3892 / \mathrm{mmr} .2021 .11900$

\begin{abstract}
Colorectal cancer (CRC), one of the most common cancer types, causes a large number of cancer-related mortalities annually worldwide. Dysregulated microRNAs (miRNAs/miR) are closely associated with the malignant progression of CRC. Therefore, the present study aimed to investigate the expression and regulatory role of miR-592 in CRC. It was found that miR-592 expression was significantly elevated in CRC tissues and cell lines, and was associated with the prognosis of patients. Cellular phenotype assays demonstrated that miR-592 could promote CRC cell proliferation, migration and invasion. Bioinformatics analysis demonstrated that miR-592 mainly participated in the positive regulation of transcription, as well as the regulation of cell motility. Moreover, miR-592 targets were enriched in several signaling pathways, such as the 'mTOR' and 'FoxO' signaling pathways. In addition, secreted protein acidic and rich in cysteine (SPARC) was identified as a target of miR-592 in CRC. The present results suggested that miR-592 acts as an oncogene in CRC, in part, by directly inhibiting SPARC expression. Collectively, the present study provides a novel potential therapeutic strategy for CRC.
\end{abstract}

\section{Introduction}

Colorectal cancer (CRC) is one of the most common malignant tumors, leading to $\sim 700,000$ cancer-related mortalities each year worldwide (1). In China, the International Agency for Research on Cancer reported that CRC accounts for $6.3 \%$ of all malignancy-related mortalities in 2012 (2). Despite advances in therapeutic methods, almost half of patients with CRC still experience tumor metastasis or recurrence after treatment, which causes a poor overall survival (OS) (3). Therefore, elucidation of

Correspondence to: Dr Chengcheng Gao, Department of Gastroenterology, The Affiliated Huai'an No. 1 People's Hospital of Nanjing Medical University, 6 Beijing West Road, Huaiyin, Huai'an, Jiangsu 223300, P.R. China

E-mail: gcc3501879@163.com

Key words: microRNA-592, colorectal cancer, secreted protein acidic and rich in cysteine, proliferation, metastasis the potential mechanisms of CRC proliferation and metastasis is important for improving the treatment and prognosis of patients with CRC.

MicroRNAs (miRNAs/miRs) are a class of small non-coding RNAs (18-25 nucleotides in length) that act as post-transcriptional regulators of gene expression by direct binding to the 3' untranslated regions (3'UTRs) of target mRNAs; this binding induces mRNA degradation and/or translational repression (4). miRNAs participate in a number of biological processes, such as cell cycle progression, proliferation, invasion, migration and cell metabolism (5-8). The regulatory role of miR-592 is context-dependent. For instance, miR-592 functions as a tumor suppressor in breast cancer (9), non-small cell lung cancer (10) and glioma (11), but as an oncogene in gastric cancer (12). Moreover, contrary results have been observed in CRC. $\mathrm{Fu}$ et al (13) reported an oncogenic role of miR-592 in CRC by targeting forkhead box O3A (FoxO3A). However, Liu et al (14) revealed that miR-592 can inhibit the proliferation of CRC cells by suppressing cyclin D3 expression.

Therefore, the present study aimed to further verify miR-592 expression and investigate its regulatory mechanism in CRC.

\section{Materials and methods}

Clinical samples. A total of 35 paired CRC tissues and adjacent normal tissues (ANTs) were collected from patients who underwent surgical resection at The Affiliated Huai'an No. 1 People's Hospital of Nanjing Medical University (Huai'an, China) between January 2017 and June 2019. The healthy tissue was $>2 \mathrm{~cm}$ away from the CRC tissue. All samples were confirmed by three pathologists independently and stored at $-80^{\circ} \mathrm{C}$ until use. The inclusion criteria for the patients to be enrolled in the study were as follows: i) Patients with CRC whose histologic slides were identified by two independent pathologists; and ii) patients with CRC who had not been treated before surgery, such as chemoradiotherapy. Otherwise, patients were excluded. The age of patients with CRC ranged between 43 and 76 years old. Written informed consent was obtained from each participant, and the study was approved by the Ethics Committees of The Affiliated Huai'an No. 1 People's Hospital of Nanjing Medical University.

Cell culture and transfection. The normal colonic epithelial cell (FHC) and CRC cell lines (HCT8, HT29, HCT116, SW480 
and SW620) were obtained from The Cell Bank of Type Culture Collection of The Chinese Academy of Sciences. Cells were cultured in DMEM that was supplemented with 10\% FBS (both from Invitrogen; Thermo Fisher Scientific, Inc.), $100 \mathrm{U} / \mathrm{ml}$ penicillin and $100 \mu \mathrm{g} / \mathrm{ml}$ streptomycin in a humidified atmosphere containing $5 \% \mathrm{CO}_{2}$ at $37^{\circ} \mathrm{C}$. All cells had been authenticated using short tandem repeat profiling. As conducted in previous studies $(15,16)$, the present study performed subsequent experiments using two cancer cells (HT29 and SW480).

The miR-592 inhibitor and negative control (NC) were designed by Shanghai GenePharma Co., Ltd., and their corresponding core sequences were as follows: miR-592 inhibitor, 5'-ACATCATCGCATATTGACACAA-3' and NC, 5'-TTCTCC GAACGTGTCACGTTTC-3'. The small interfering (si)RNA of secreted protein acidic and rich in cysteine (SPARC; siSPARC) was generated from Guangzhou Ribobio Co., Ltd., and the corresponding sequences were 5'-AACAAGACCUU CGACUCUUCC-3' (siSPARC) and 5'-GCUCACAGCUCAAU CCUAAUC-3' (siNC). Both miR-NC and siNC were non-targeting. The transfection was conducted at a concentration of $100 \mathrm{nM}$ using Lipofectamine ${ }^{\circledR} 2000$ (Invitrogen; Thermo Fisher Scientific, Inc.) at $37^{\circ} \mathrm{C}$ for $6 \mathrm{~h}$ according to the manufacturer's protocol. After transfection for $48 \mathrm{~h}$, subsequent experimentations were conducted.

$R N A$ extraction and reverse transcription-quantitative (RT- $q)$ $P C R$. Total RNA was isolated using TRIzol ${ }^{\circledR}$ reagent (Invitrogen; Thermo Fisher Scientific, Inc.) according to the manufacturer's instruction. RT-qPCR of miR-592 was performed using a Hairpin-it ${ }^{\text {TM }}$ miRNA Normalization RT-PCR Quantitation kit (Shanghai GenePharma Co., Ltd.), and U6 snRNA was regarded as the internal control. The following thermocycling conditions of miRNA RT-qPCR were as follows: Initial denaturation at $95^{\circ} \mathrm{C}$ for $3 \mathrm{~min}, 40$ cycles of denaturation at $95^{\circ} \mathrm{C}$ for $15 \mathrm{sec}$, annealing and elongation at $62^{\circ} \mathrm{C}$ for $34 \mathrm{sec}$. The RT-qPCR primer sequences were as follows: miR-592 forward, 5'-ACGTTGTGTCAATATGCGATGA-3' and reverse, 5'-GTG CAGGGTCCGAGGT-3'; and U6 forward, 5'-CTCGCTTCGG CAGCACA-3' and reverse, 5'-AACGCTTCACGAATTTG CGT-3'. cDNA was synthesized using the PrimeScript RT-PCR kit (Takara Biotechnology Co., Ltd.), and RT-qPCR for SPARC transcript was performed in $20 \mu 1$ reactions using the TB Green ${ }^{\circledR}$ Fast qPCR mix kit (Takara Biotechnology Co., Ltd.). GAPDH was used as the internal control. The following thermocycling conditions of mRNA RT-qPCR were as follows: Initial denaturation at $95^{\circ} \mathrm{C}$ for $5 \mathrm{~min}, 40$ cycles of denaturation at $95^{\circ} \mathrm{C}$ for $10 \mathrm{sec}$, annealing at $60^{\circ} \mathrm{C}$ for $30 \mathrm{sec}$, and elongation at $72^{\circ} \mathrm{C}$ for $30 \mathrm{sec}$. The primers used were as follows: SPARC forward, 5'-GTGCAGAGGAAACCGAAGAG-3' and reverse, 5'-AGTGGCAGGAAGAGTCGAAG-3'; and GAPDH forward, 5'-GAGTCAACGGATTTGGTCGT-3' and reverse, 5'-TTGATT TTGGAGGGATCTCG-3'. Relative miR-592 expression was measured using the $2^{-\Delta \Delta \mathrm{Cq}}$ method (17).

Immunoblotting analysis. CRC cells were washed and lysed in RIPA lysis buffer (Nanjing KeyGen Biotech Co., Ltd.). Subsequently, the protein was determined using a BCA protein content detection kit (Nanjing KeyGen Biotech Co., Ltd.) and boiled for $5 \mathrm{~min}$. Denatured protein $(10 \mu \mathrm{g} / \mathrm{lane})$ was separated via SDS-PAGE on $10 \%$ gel, and then transferred to PVDF membranes. The membranes were blocked with TBS with Tween-20 (0.1\%) containing 5\% skimmed milk at room temperature for $1 \mathrm{~h}$ and subsequently incubated with primary antibodies (rabbit polyclonal SPARC antibody, 1:1,000, cat. no. 15274-1-AP, ProteinTech Group, Inc.; and rabbit polyclonal GAPDH antibody, 1:5,000, cat. no. 10494-1-AP, ProteinTech Group, Inc.) overnight at $4^{\circ} \mathrm{C}$. The membranes were then incubated with anti-IgG secondary antibodies conjugated to horseradish peroxidase at room temperature for $1 \mathrm{~h}$ [HRP-conjugated Goat anti-rabbit IgG (H+L); 1:5,000; cat. no. SA00001-2; ProteinTech Group, Inc.]. Bands were visualized using the ECL reagent (Nanjing KeyGen Biotech Co., Ltd.).

Plasmid construction and luciferase reporter assay. Both wild-type (Wt) and mutant (Mut) SPARC 3'UTRs were amplified via PCR and cloned into the pMIR-Report plasmid (Ambion; Thermo Fisher Scientific, Inc.). Wt 3'UTRs were achieved from gDNA and Mut 3'UTRs were chemically synthesized (Shanghai GeneChem Co., Ltd.). Taq Plus DNA polymerase (Vazyme Biotech Co., Ltd.) were used in PCR. The primers used were as follows: Forward, 5'-GAAACTGCCTTCCTGGGTGA-3' and reverse, 5'-GGAACCATACACTCCCTGTGT-3'. The thermocycling conditions were as follows: Initial denaturation at $98^{\circ} \mathrm{C}$ for $5 \mathrm{~min}, 40$ cycles of denaturation at $98^{\circ} \mathrm{C}$ for $10 \mathrm{sec}$, annealing at $60^{\circ} \mathrm{C}$ for $30 \mathrm{sec}$, and elongation at $72^{\circ} \mathrm{C}$ for $1 \mathrm{~min}$. For the luciferase assay, 293T cells (The Cell Bank of Type Culture Collection of The Chinese Academy of Sciences) were cultured in 24-well plates and co-transfected with plasmids along with miR-592 inhibitor $(100 \mathrm{nM})$ or $\mathrm{NC}(100 \mathrm{nM})$ at $37^{\circ} \mathrm{C}$ for $48 \mathrm{~h}$. Transfection was conducted using Lipofectamine ${ }^{\circledR} 2000$ (Invitrogen; Thermo Fisher Scientific, Inc.). Cells were collected after $48 \mathrm{~h}$, and the luciferase reporter activity was analyzed using a Dual luciferase Reporter assay system (Promega Corporation) in which Renilla luciferase was used for normalization.

Cell Counting Kit (CCK)- 8 assay. The proliferative ability of cells was measured using the CCK-8 assay (Nanjing KeyGen Biotech Co., Ltd.), according to the manufacturer's instructions. Briefly, HT29 and SW480 cells were plated into a 96-well plate at a density of $1.0 \times 10^{3}$ cells/well and incubated at $37^{\circ} \mathrm{C}$ for 24 , 48 and $72 \mathrm{~h}$. Then, the absorbance was measured at $450 \mathrm{~nm}$ on a microplate reader (Infinite M200 PRO; Tecan Group, Ltd.).

A 5-Ethynyl-2'-deoxyuridine (EdU) incorporation assay. The EdU incorporation assay was conducted using an EdU DNA Cell Proliferation kit (Guangzhou RiboBio Co., Ltd.). After incubation with EdU (1X) $37^{\circ} \mathrm{C}$ for $2 \mathrm{~h}, \mathrm{HT} 29$ and SW480 cells were fixed with paraformaldehyde (4\%) at room temperature for 30 min followed by staining with Apollo Dye Solution at room temperature for $30 \mathrm{~min}$. Then, the cells were mounted with Hoechst (1X) at room temperature for $30 \mathrm{~min}$. Finally, cells were imaged and counted using an Olympus FSX100 fluorescence microscope (Olympus Corporation) at x200 magnification.

Migration assay. HT29 and SW480 cells were seeded into 6-well plates in DMEM without FBS and grown until $90 \%$ confluency for $24 \mathrm{~h}$. Then, the cells were scratched using 200- $\mu \mathrm{l}$ pipette tips and washed twice with PBS. Next, cells were cultured in an incubator containing $5 \% \mathrm{CO}_{2}$ at $37^{\circ} \mathrm{C}$. Images were captured after 0 and $24 \mathrm{~h}$ under an Olympus FSX100 light microscope 
(Olympus Corporation) at x 200 magnification. Images were subsequently analyzed by ImageJ software (v1.53; National Institutes of Health).

Transwell invasion assay. The top chambers of Transwell chambers (8- $\mu \mathrm{m}$ pore size; BD Biosciences) were coated with Matrigel mix (BD Biosciences) at $37^{\circ} \mathrm{C}$ for $2 \mathrm{~h}$ and $1.5 \times 10^{5}$ cells were seeded on the upper chamber containing $100 \mu 1$ DMEM without FBS. Then, $500 \mu 1 \mathrm{DMEM}$ with $10 \% \mathrm{FBS}$ was added to the bottom chamber. After being cultured at $37^{\circ} \mathrm{C}$ for $24 \mathrm{~h}$, cells that passed through the Matrigel were fixed with $100 \%$ methanol at room temperature for $20 \mathrm{~min}$ and stained with crystal violet $(0.1 \%)$ at room temperature for $30 \mathrm{~min}$. Finally, cells were counted under an Olympus FSX100 light microscope (Olympus Corporation) at x200 magnification.

Immunohistochemistry analysis. The tissue samples were fixed with $4 \%$ paraformaldehyde at room temperature for $24 \mathrm{~h}$ and embedded in paraffin. Then, slices (5 $\mu \mathrm{m}$ thickness) were washed twice with phosphate buffered saline at room temperature for 5 min each time and incubated with $10 \%$ goat serum (Invitrogen; Thermo Fisher Scientific, Inc.) at $37^{\circ} \mathrm{C}$ for $30 \mathrm{~min}$. Immunohistochemistry analysis was performed on paraffin-embedded sections using a primary antibody against SPARC (1:1,000; cat. no. HPA003020; Sigma-Aldrich; Merck $\mathrm{KGaA}$ ) overnight at $4^{\circ} \mathrm{C}$. After being washed three times, sections were incubated with a horseradish peroxidase-conjugated IgG (1:1,000; cat. no. ab7090; Abcam) at room temperature for $1 \mathrm{~h}$. In total, three high power fields (magnification, $\mathrm{x} 400$ ) were randomly selected from CRC tissues using an Olympus FSX100 light microscope (Olympus Corporation). For histological scoring, the degree of positivity was initially classified according to scoring both the proportion of positively stained tumor cells and the staining intensities. Scores representing the proportion of positively stained tumor cells were graded as follows: i) $0, \leq 10 \%$; ii) $1,11-25 \%$; iii) $2,26-50 \%$; iv) $3,51-75 \%$; and v) $4,>75 \%$. The intensity of staining was determined as follows: i) 0 , no staining; ii) 1 , weak staining (light yellow); iii) 2 , moderate staining (yellow brown); and iv) 3, strong staining (brown). The reactivity degree was assessed by $\geq 2$ pathologists independently.

Immunofluorescence analysis. For the immunofluorescence assay, cells were fixed in $4 \%$ paraformaldehyde at room temperature for $20 \mathrm{~min}$, permeabilized using $0.5 \%$ Triton X-100 at room temperature for $20 \mathrm{~min}$, blocked with $5 \%$ goat serum at room temperature for $2 \mathrm{~h}$, and incubated with a primary antibody against SPARC (1:1,000; cat. no. HPA003020; Sigma-Aldrich; Merck KGaA) overnight at $4^{\circ} \mathrm{C}$. After being washed three times, cells were incubated with a secondary antibody (1:500; cat. no. ab150081; Abcam) at room temperature for $1 \mathrm{~h}$. Coverslips were counterstained with DAPI at room temperature for 5 min and imaged with a confocal laser scanning microscope (Olympus FV1000; Olympus Corporation) at x400 magnification.

Bioinformatics analysis. The differentially expressed miRNAs and mRNAs in CRC deposited in the Gene Expression Omnibus (GEO) database (https://www.ncbi.nlm.nih.gov/geo/) were analyzed using the online tool GEO2R (https://www.ncbi.nlm. nih.gov/geo/geo2r/). Three GEO datasets for miRNA expression in CRC (GSE128446, GSE126093 and GSE125961) (18) were found using the key words 'microRNA' and 'colorectal cancer'. The putative targets of miR-592 were identified using the TargetScan (v7.2) database (http://www.targetscan. org/vert_72/) and the miRDB database (http://mirdb.org). Gene Ontology (GO) (19) and Kyoto Encyclopedia of Genes and Genomes (KEGG) (18) analyses were conducted on the Database for Annotation, Visualization and Integrated Discovery website (https://david.ncifcrf.gov). The protein-protein interaction (PPI) network was constructed using Search Tool for the Retrieval of Interacting Genes/Proteins (https://string-db.org) and Cytoscape (v3.8.2; https://cytoscape.org). Analysis of functional modules and hub genes were explored using the MCODE and Cytohubba tools in Cytoscape. The expression and location of SPARC protein in CRC cells (RH-30) revealed by immunohistochemistry and immunofluorescence were further examined using The Human Protein Atlas (https://www.proteinatlas.org) database (20).

Statistical analysis. Data were obtained from three independent experiments and are presented as the mean \pm SD. Statistical analysis of the difference between groups was performed using SPSS 22.0 (IBM, Corp.) or GraphPad Prism 8.0 (GraphPad Software, Inc.) using the Student's paired or unpaired t-test or one-way ANOVA, followed by Tukey's post hoc test. The association between miR-592 expression and the clinicopathological characteristics of patients with CRC was assessed using the $\chi^{2}$ test. The correlation between miR-592 expression and SPARC expression was assessed via Pearson correlation coefficients. The Kaplan-Meier method with the log-rank test was used to calculate the OS rate. $\mathrm{P}<0.05$ was considered to indicate a statistically significant difference.

\section{Results}

miR-592 expression is significantly elevated in CRC and is associated with the prognosis of patients. The GEO database was first used to investigate the expression level of miR-592 in CRC. In total, three GEO datasets for miRNA expression in CRC (GSE128446, GSE126093, GSE125961) (18) were found using the key words 'microRNA' and 'colorectal cancer'. The characteristics of these GEO datasets are listed in Table I. As presented in Fig. 1A-C, miR-592 expression was significantly upregulated in CRC tissues compared with that in healthy tissues. Moreover, RT-qPCR analysis demonstrated that miR-592 expression was significantly elevated in CRC tissues and cell lines compared with normal tissues and FHC cells, respectively (Fig. 1D and E). The expression of miR-592 in lymphatic metastatic nodules was higher compared with that in primary tumor sites (Fig. 1D).

To assess the association between miR-592 expression and the clinicopathologic characteristics of patients with CRC, patients were categorized into the miR-592 high group $(n=18)$ and low group $(n=17)$ according to the median value of miR-592 expression. As presented in Table II, miR-592 expression was significantly associated with TNM stage $(\mathrm{P}=0.018)$ and lymphatic metastasis $(\mathrm{P}=0.041)$. Furthermore, Kaplan-Meier survival analysis indicated that patients with CRC with higher miR-592 expression may have shorter OS times (hazard ratio $=2.3 ; \mathrm{P}=0.047$ ) (Fig. $1 \mathrm{~F})$. 
Table I. Detailed characteristics of Gene Expression Omnibus datasets enrolled in this study.

\begin{tabular}{lllll}
\hline $\begin{array}{l}\text { Characteristics } \\
\text { Organism }\end{array}$ & \multicolumn{1}{c}{$\begin{array}{c}\text { GSE128446 } \\
\text { Homo sapiens }\end{array}$} & \multicolumn{1}{c}{$\begin{array}{c}\text { GSE126093 } \\
\text { Homo sapiens }\end{array}$} & \multicolumn{1}{c}{$\begin{array}{c}\text { GSE125961 } \\
\text { Homo sapiens }\end{array}$} & \multicolumn{1}{c}{$\begin{array}{c}\text { GSE126092 } \\
\text { Homo sapiens }\end{array}$} \\
\hline Experiment type & $\begin{array}{l}\text { Non-coding RNA } \\
\text { profiling by array }\end{array}$ & $\begin{array}{l}\text { Non-coding RNA } \\
\text { profiling by array }\end{array}$ & $\begin{array}{l}\text { Non-coding RNA profiling by } \\
\text { high throughput sequencing }\end{array}$ & $\begin{array}{l}\text { Expression profiling } \\
\text { by array }\end{array}$ \\
Platform & GPL14767 & GPL18058 & GPL16791 & GPL121047 \\
Sample size & Four normal tissues & 10 paired ANTs & 10 paired ANTs and CRC & 10 paired ANTs \\
& and 18 CRC tissues & and CRC tissues & tissues & and CRC tissues \\
BioProject & PRJNA527778 & PRJNA521013 & PRJNA518096 & PRJNA521015 \\
\hline
\end{tabular}

CRC, colorectal cancer; ANTs, adjacent normal tissues.

miR-592 knockdown suppresses the proliferation, migration and metastasis of CRC cells. To examine the biological role of miR-490-3p in CRC, HT29 and SW480 cells were transfected with miR-592 inhibitor and NC. RT-qPCR analysis was used to confirm the transfection efficiency (Fig. 2A). The CCK-8 and EdU incorporation assays demonstrated that miR-592 knockdown significantly inhibited the proliferative ability of CRC cells (Fig. 2B and C). In addition, the wound healing assay identified that, compared with $\mathrm{NC}$ transfection, miR-592 inhibitor transfection significantly decreased cell migration (Fig. 2D). The Transwell invasion assay also demonstrated that the invasive ability of CRC cells transfected with inhibitor was significantly decreased compared with that of cells transfected with NC (Fig. 2E).

Analysis of putative targets of miR-592. To identify the targets of miR-592, the current study first overlapped predicted results searched in the TargetScan and miRDB algorithms, and 323 putative targets were found (Fig. 3A). GO analysis of these targets indicated that miR-592 participated in various biological processes, such as 'positive regulation of transcription', 'extracellular matrix organization' and 'regulation of cell motility' (Fig. 3B). KEGG analysis identified that the targets of miR-592 were significantly associated with several signaling pathways, including the 'ErbB signaling pathway', 'mTOR signaling pathway' and 'FoxO signaling pathway' (Fig. 3C). In addition, a PPI network of miR-592 targets was constructed, among which five functional modules were discovered using the MCODE tool (Fig. 3D). Cytohubba analysis revealed seven hub genes among the PPI network (Fig. 3E).

It has been reported that one miRNA may regulate the expression of multiple genes and vice versa (21). Considering the upregulation of miR-592 in CRC, it was suggested that these targets with downregulated expression in CRC may be regulated by miR-592. It was found that SPARC, one of the hub genes, was significantly downregulated in CRC tissues compared with that in ANTs based on GSE126092 analysis (Fig. 3G). However, the expression of the other six hub genes was either upregulated or undifferentiated in CRC tissues compared with ANTs (data not shown). Additionally, SPARC belonged to a functional module in the PPI network (Fig. 3F). Immunohistochemistry analysis demonstrated that SPARC was barely expressed in CRC cells but was highly enriched in the tumor microenvironment (Fig. 3H). In addition, immunofluorescence analysis
Table II. Associations between the clinicopathological characteristics of patients with colorectal cancer and miR-592 expression levels.

\begin{tabular}{|c|c|c|c|c|}
\hline \multirow[b]{2}{*}{ Characteristics } & \multirow[b]{2}{*}{ Number } & \multicolumn{2}{|c|}{$\begin{array}{l}\text { miR-592 } \\
\text { expression }\end{array}$} & \multirow[b]{2}{*}{ P-value } \\
\hline & & High & Low & \\
\hline \multicolumn{5}{|l|}{ Sex } \\
\hline Male & 24 & 13 & 11 & \\
\hline Female & 11 & 5 & 6 & 0.730 \\
\hline \multicolumn{5}{|l|}{ Age, year } \\
\hline$>60$ & 23 & 12 & 11 & \\
\hline$\leq 60$ & 12 & 6 & 6 & 0.900 \\
\hline \multicolumn{5}{|l|}{ TNM stage } \\
\hline $\mathrm{I}+\mathrm{II}$ & 17 & 5 & 12 & \\
\hline $\mathrm{III}+\mathrm{IV}$ & 18 & 13 & 5 & 0.018 \\
\hline \multicolumn{5}{|l|}{ Histology grade } \\
\hline Well & 12 & 5 & 7 & \\
\hline Moderate + poor & 23 & 13 & 10 & 0.320 \\
\hline \multicolumn{5}{|c|}{ Lymphatic metastasis } \\
\hline No & 20 & 8 & 12 & \\
\hline Yes & 15 & 10 & 5 & 0.041 \\
\hline \multicolumn{5}{|l|}{ Tumor size, $\mathrm{cm}$} \\
\hline$\leq 4.5$ & 19 & 7 & 12 & \\
\hline$>4.5$ & 16 & 11 & 5 & 0.092 \\
\hline
\end{tabular}

identified that SPARC was mainly located in the vesicles of RH-30 tumor cells (Fig. 3I).

SPARC is a direct target of miR-592 in CRC. To identify SPARC as the direct target of miR-592 in CRC, its expression was firstly evaluated. RT-qPCR analysis demonstrated that SPARC expression was significantly downregulated in CRC tissues and cell lines compared with ANTs and FHC cells, respectively (Fig. 4A and B). Furthermore, a weak negative correlation was identified between miR-592 expression and 
A

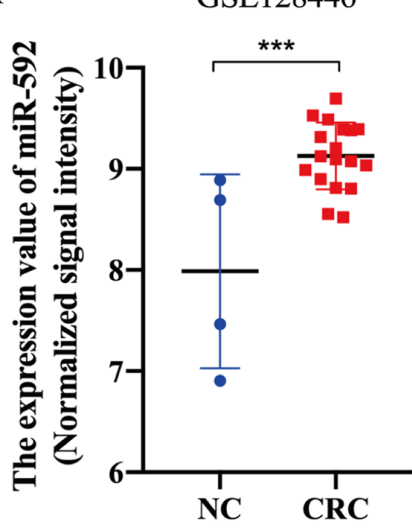

B

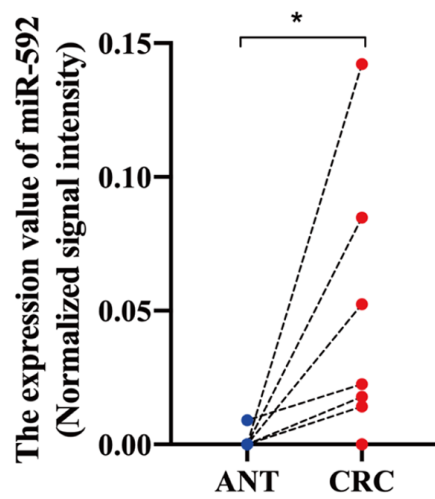

C

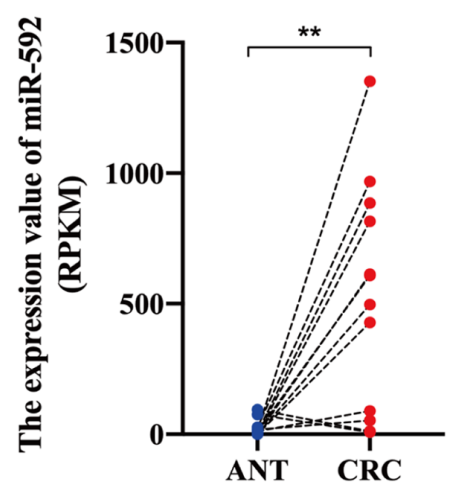

D

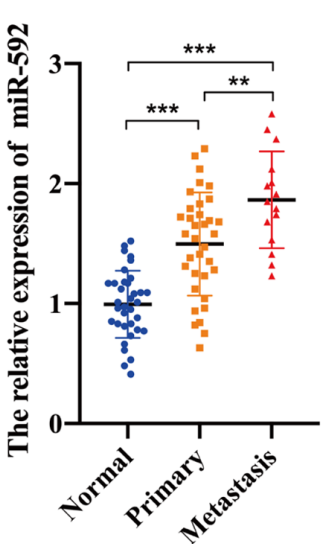

E

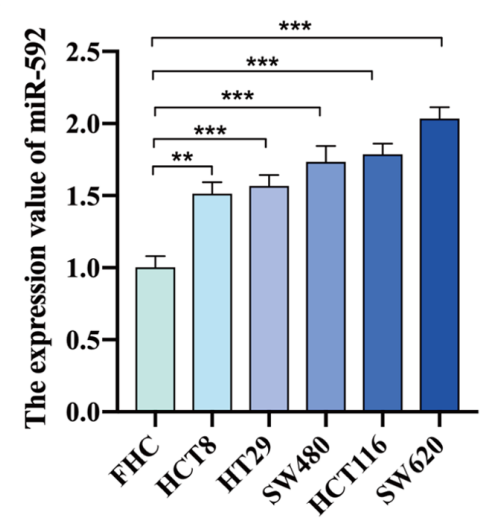

F

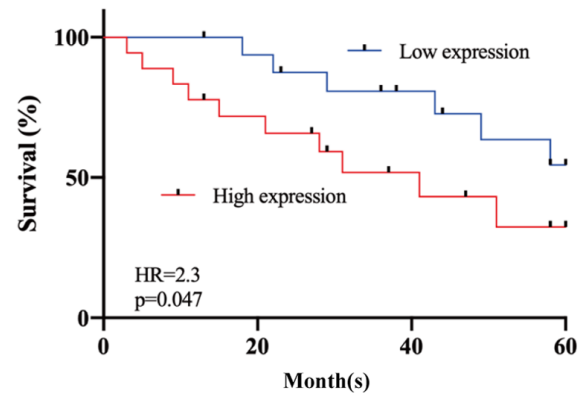

Figure 1. miR-592 expression is significantly upregulated in CRC and is associated with the prognosis of patients. Expression level of miR-592 in CRC tissues and normal tissues based on analysis of (A) GSE128446, (B) GSE126093 and (C) GSE125961. (D) miR-592 expression in clinical normal tissues, primary CRC tissues and metastatic nodules. (E) Expression of miR-592 in CRC cell lines and FHC cells. (F) Kaplan-Meier curve analysis of patients with CRC. * $<0.05$, ${ }^{* *} \mathrm{P}<0.01,{ }^{* * * *} \mathrm{P}<0.001$. NC, negative control; CRC, colorectal cancer; ANT, adjacent normal tissues; miR, microRNA; HR, hazard ratio; RPKM, Reads Per Kilobase per Million.

SPARC expression in CRC tissues (Fig. 4C). When the miR-592 inhibitor was transfected into HT29 and SW480 cells, the expression of SPARC at both the transcriptional and protein levels was significantly upregulated in CRC cells (Fig. 4D and E). Moreover, the direct regulatory role of miR-592 on SPARC expression was assessed using a dual-luciferase assay, in which Renilla luciferase was used for normalization. Interestingly, the SPARC 3'UTR sequence had two binding sites of miR-592 with a high conserved score. Therefore, several luciferase reporter plasmids were constructed with Wt and Mut1-3 SPARC 3'UTRs (Fig. 4F). As presented in Fig. 4G, after co-transfecting with miR-592 inhibitor, the luciferase activity was significantly elevated in luciferase reporter groups with Wt and Mut1-2 SPARC 3'UTRs, but not in the luciferase reporter group with Mut3 SPARC 3'UTRs, which suggested that miR-592 can directly bind to two sites in SPARC 3'UTRs.

Silencing SPARC significantly reverses the effect of $m i R-592$ knockdown on CRC cells. To investigate the regulatory role of SPARC in CRC, SPARC expression was inhibited by transfecting cells with siSPARC. Western blotting results demonstrated that siSPARC effectively suppressed SPARC expression (Fig. 5A). The cellular phenotypes indicated that SPARC knockdown significantly promoted the proliferation, migration and invasion of CRC cells (Fig. 5B-E).
Subsequently, CRC cells were co-transfected with siSPARC and miR-592 inhibitor to investigate whether the oncogenic role of miR-592 was directly mediated by SPARC inhibition (Fig. 5A). The CCK-8 and EdU incorporation assays suggested that SPARC knockdown significantly reversed the effect of miR-592 knockdown on cell proliferation (Fig. 5B and C). Consistently, the migration and Transwell invasion assays identified that both the migratory and invasive abilities, which were suppressed by miR-592 knockdown, were restored after SPARC knockdown in HT29 and SW480 cells (Fig. 5D and E).

\section{Discussion}

Dysregulated miR-592 has been reported to serve a vital role in the malignant progression of multiple cancer types, such as hepatocellular carcinoma and prostate cancer (22-24). Liu et al (25) revealed that miR-592 expression was upregulated in clinical CRC tissues. Consistently, the present study demonstrated that miR-592 expression was significantly upregulated in CRC tissues and cell lines compared with ANTs and FHC cells, respectively. Furthermore, patients with CRC with higher miR-592 expression had a poorer prognosis compared with those with low expression. Fu et al (13) reported that miR-592 can promote the proliferation and metastasis of CRC cells, in part, by targeting FoxO3A. In the present study, SPARC was identified as another 
A

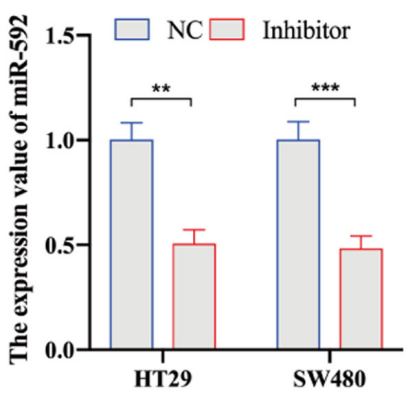

C
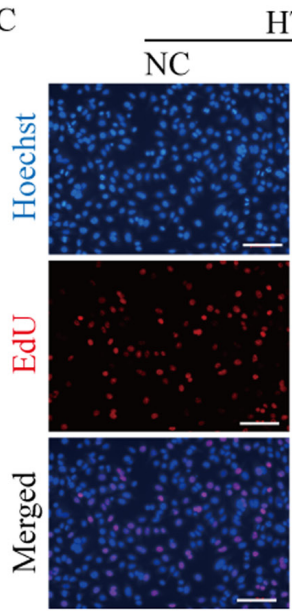

HT29

B
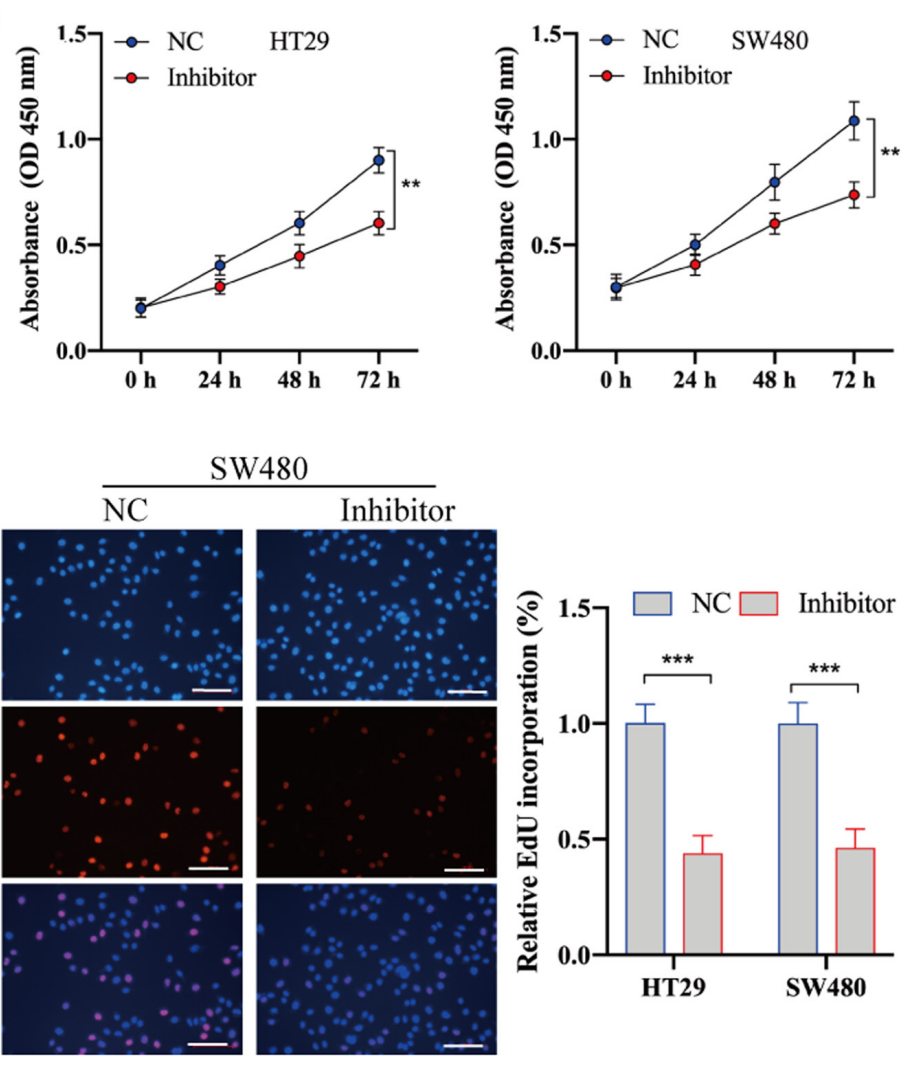

SW480
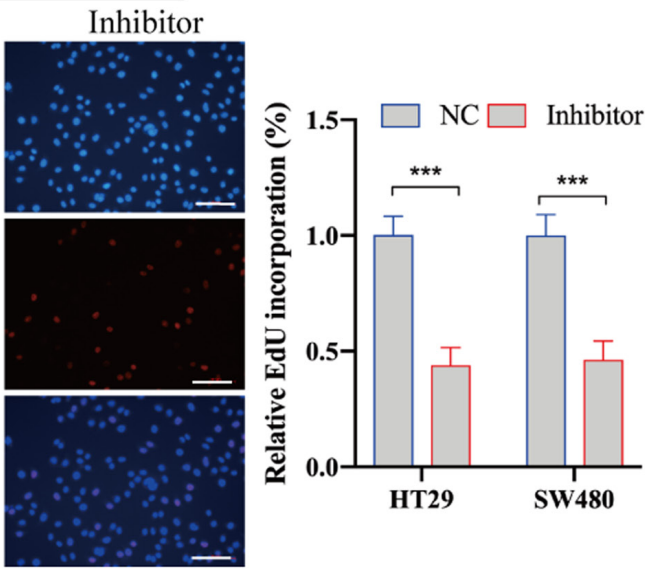

D

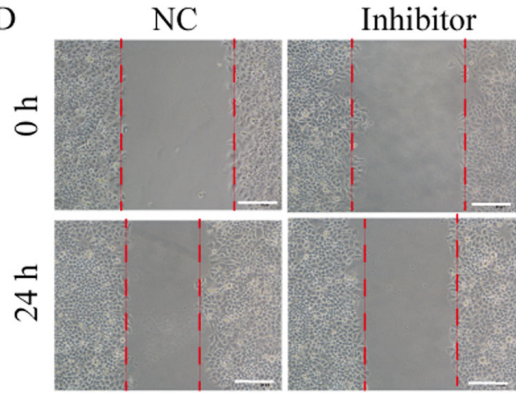

HT29

E

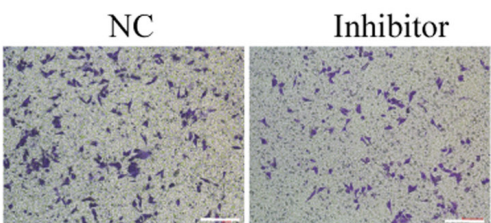

HT29

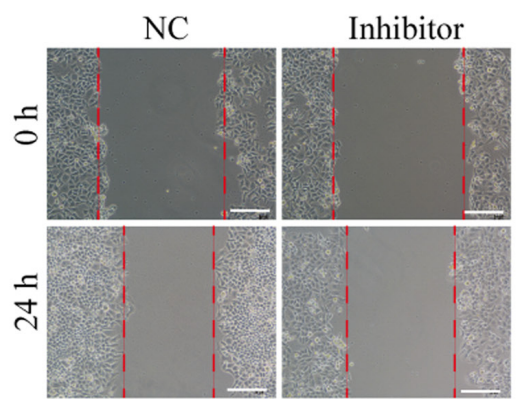

SW480

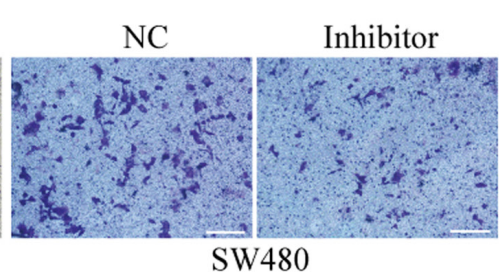

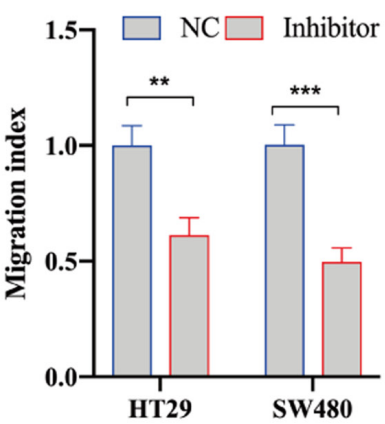

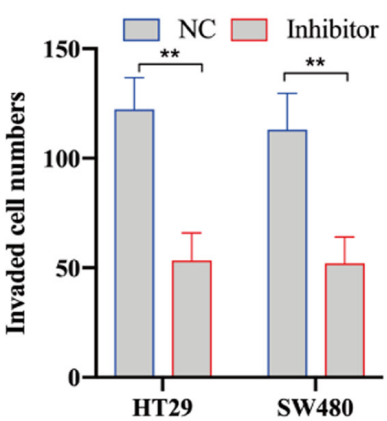

Figure 2. Knockdown of miR-592 expression suppresses the proliferation and metastasis of CRC cells. (A) Transfection efficiency of miR-592 inhibitor in CRC cells. (B) Cell Counting Kit-8 and (C) EdU incorporation assays demonstrated that miR-592 inhibitor significantly suppressed CRC cell proliferation. (D) Wound healing assay showed that miR-592 downregulation significantly inhibited CRC cell migration. (E) Transwell invasion assay showed that miR-592 knockdown significantly suppressed CRC cell invasion. Scale bar, $100 \mu \mathrm{m} .{ }^{* *} \mathrm{P}<0.01,{ }^{* * *} \mathrm{P}<0.001$. NC, negative control; CRC, colorectal cancer; OD, optical density; EdU, 5-Ethynyl-2'-deoxyuridine; miR, microRNA.

target of miR-592 in CRC. Moreover, it was found that miR-592 acted as an oncogene by directly suppressing SPARC expression in CRC. Liu et al (25) also discovered that serum miR-592 expression was elevated in patients with CRC compared with healthy individuals and was significantly decreased after radical surgery. Therefore, it was hypothesized that CRC cells may secrete intracellular miR-592 into a circulating system, which offers a novel potential diagnostic biomarker of CRC. However, 

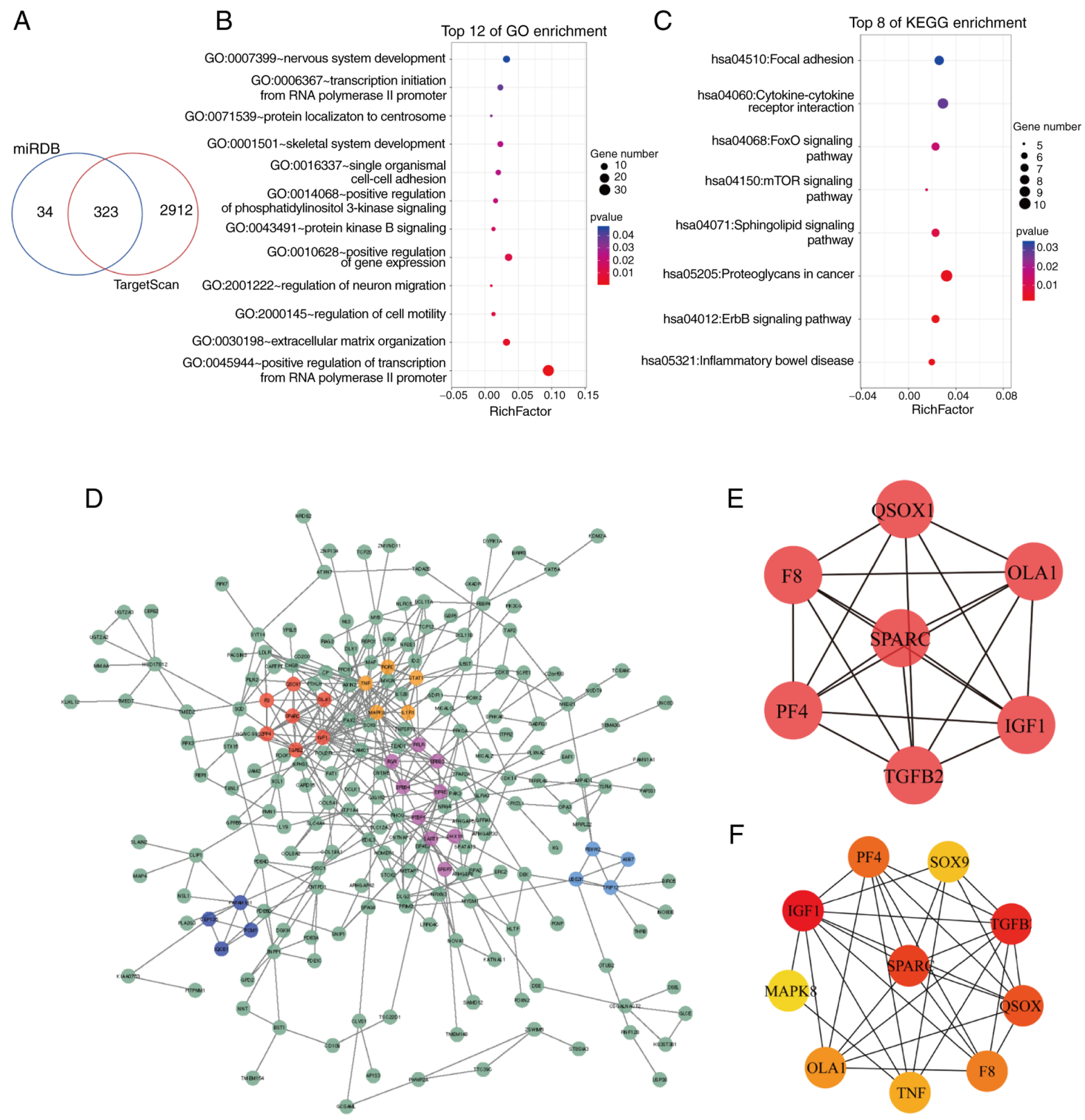

$\mathrm{F}$

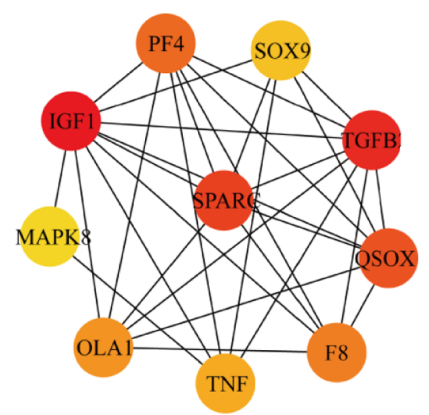

G

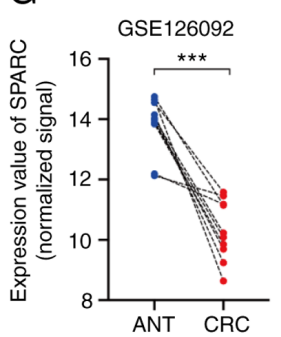

$\mathrm{H}$

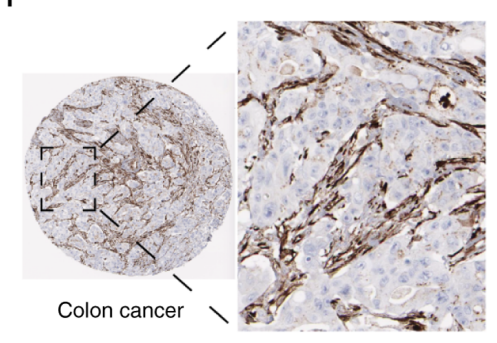

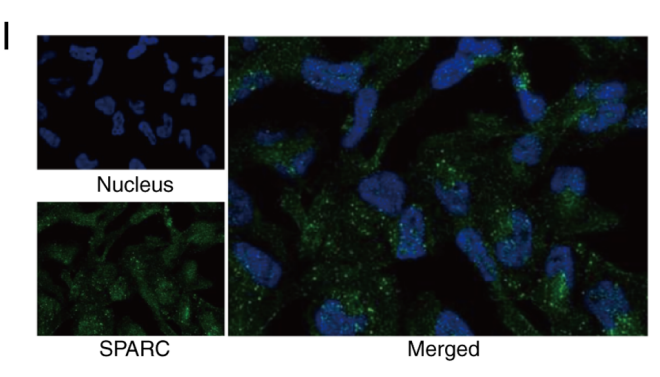

Figure 3. Bioinformatics analysis of putative targets of miR-592. (A) Putative targets of miR-592 predicted via TargetScan and miRDB. (B) GO and (C) KEGG analysis of putative targets of miR-592. (D) PPI network of miR-592 targets. (E) Hub genes in PPI network revealed by Cytohubba. (F) A functional module in the PPI network revealed by MCODE. (G) Expression level of SPARC in CRC tissues and ANTs based on analysis of GSE126092. (H) Immunohistochemistry analysis of SPARC in CRC. (I) Immunofluorescence analysis of SPARC in cells. ${ }^{* * *} \mathrm{P}<0.001$. PPI, Protein-protein interaction; CRC, colorectal cancer; miR, microRNA; ANT, adjacent normal tissues; GO, Gene Ontology; KEGG, Kyoto Encyclopedia of Genes and Genomes; SPARC, secreted protein acidic and rich in cysteine.

a contrary report regarding the low expression of miR-592 in CRC was previously published (14), and it was suggested that this phenomenon may be attributed to the heterogeneity of CRC tissues and the small sample size used in this previous study.
The promoter of SPARC has been reported to be epigenetically hypermethylated, which results in decreased SPARC expression in CRC (26). The present study demonstrated that SPARC expression was also controlled by miRNA at the 

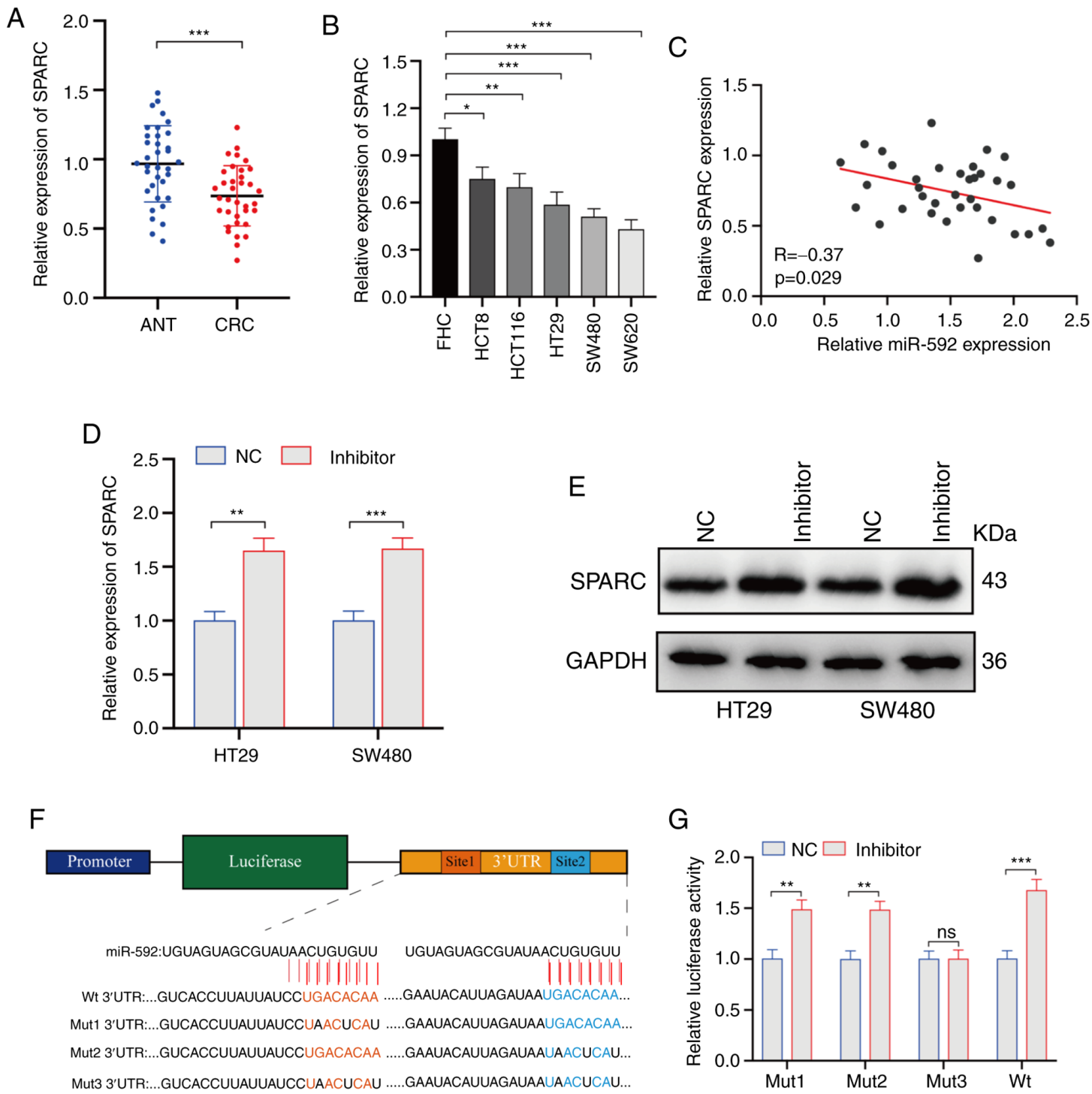

Figure 4. Identifying SPARC as a direct target of miR-592 in CRC. (A) Expression of miR-592 in clinical CRC tissues and ANTs. (B) Expression of miR-592 in CRC cell lines and FHC cells. (C) Correlation between miR-592 expression and SPARC expression in CRC tissues. (D) Transfection effect of the miR-592 inhibitor on SPARC expression in CRC cells, (E) as determined via western blotting. (F) Construction of luciferase reporter plasmids with Wt or Mut binding sites. (G) Luciferase activity of luciferase reporter plasmids co-transfected with miR-592 inhibitor or NC. ${ }^{*} \mathrm{P}<0.05,{ }^{* * *} \mathrm{P}<0.01,{ }^{* * * *} \mathrm{P}<0.001$. NC, negative control; CRC, colorectal cancer; Wt, wild-type; Mut, mutant; UTR, untranslated region; ANT, adjacent normal tissues; miR, microRNA; SPARC, secreted protein acidic and rich in cysteine; ns, not significant.

post-transcription level. Furthermore, the immunohistochemistry analysis identified that SPARC was highly enriched in the tumor microenvironment. In line with the current findings, SPARC was previously found to be upregulated in the tumor stroma, and fibroblast-derived SPARC can promote the invasiveness of CRC cells (27). Interestingly, the immunofluorescence analysis indicated that SPARC was mainly located in the vesicles of RH-30 tumor cells. Emerging evidence has revealed that cancer cells can release cellular RNAs into peripheral blood through vesicles, such as exosomes $(28,29)$. Therefore, it was hypothesized that SPARC may also be located in the vesicles of CRC cells and that the downregulation of SPARC in CRC cells may also be attributed to their enrichment in secretory vehicles.

There are several limitations in the present study. First, the number of clinical samples was relatively small. Second, the current study did not further investigate the expression or regulatory role of miR-592 and SPARC in the tumor microenvironment. Third, the association between SPARC and secretory vehicles in CRC will be examined in future studies.

In conclusion, the present findings demonstrated that miR-592 expression was significantly upregulated in CRC, and that miR-592 overexpression contributed to the promotion of cell proliferation, migration and metastasis by directly suppressing SPARC expression, which provides a novel potential therapeutic target for patients with CRC.

\section{Acknowledgements}

Not applicable.

\section{Funding}

No funding was received. 

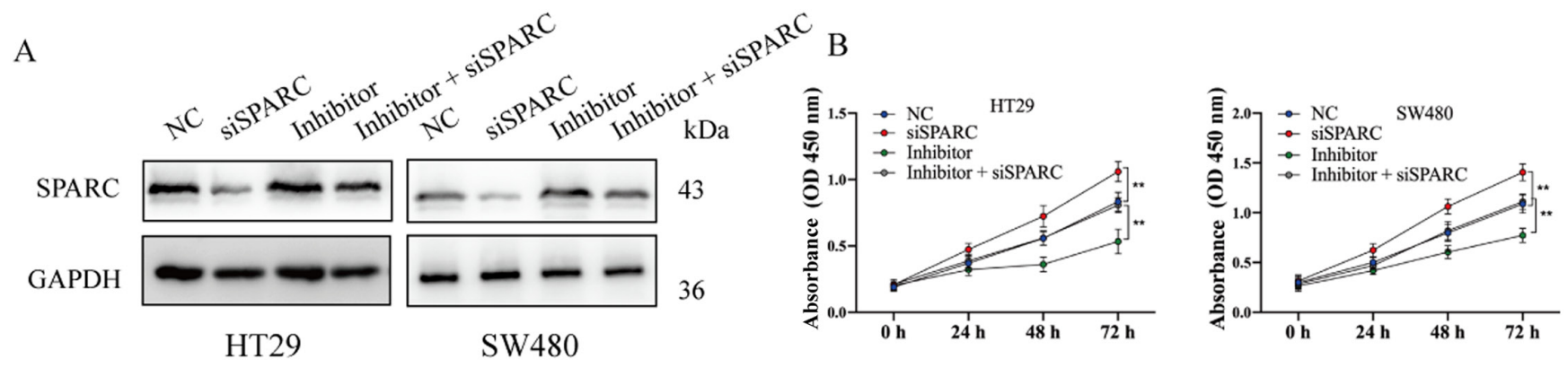

C
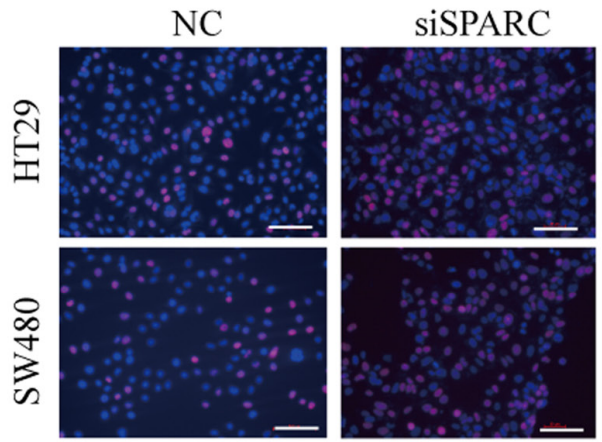

Inhibitor
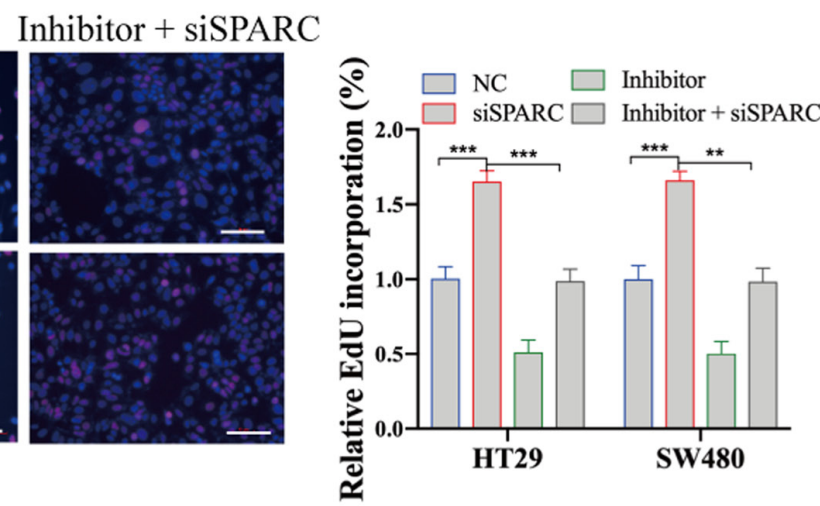

D

$\mathrm{NC}$
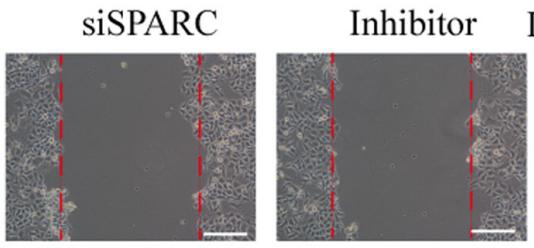

Inhibitor + siSPARC

$0 \mathrm{~h}$
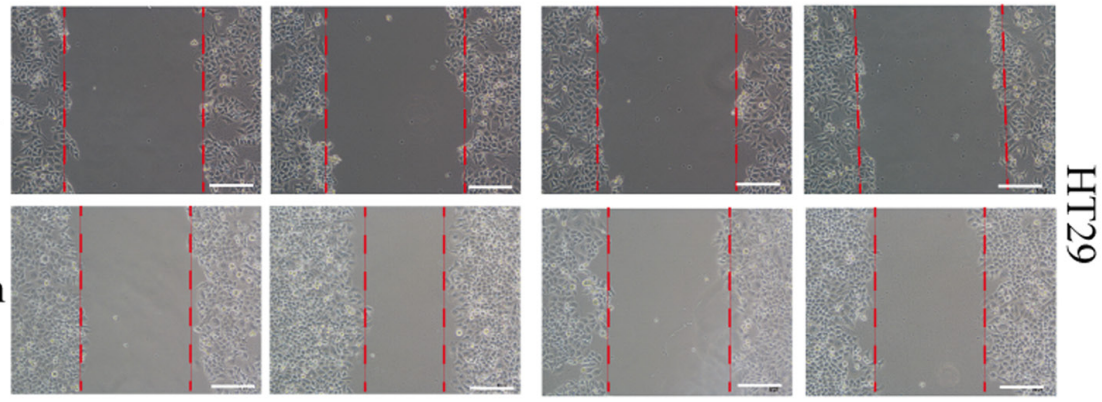

$24 \mathrm{~h}$
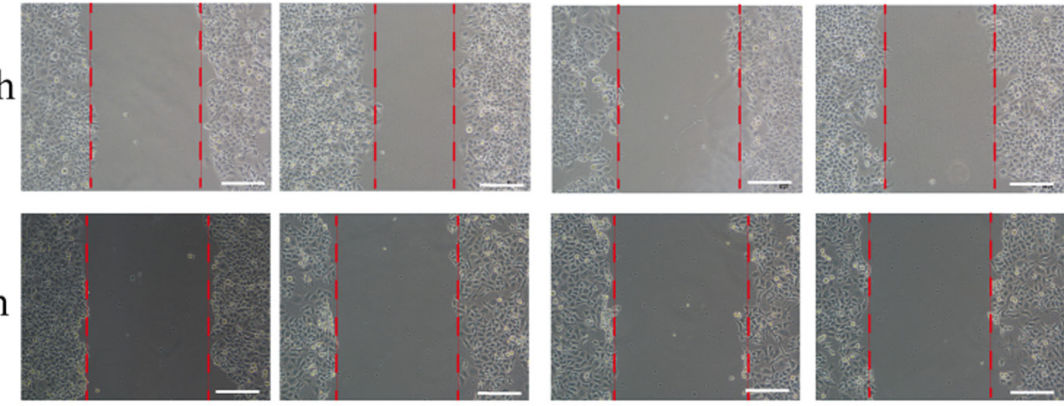

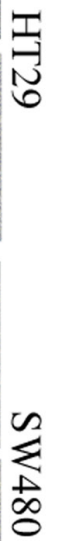

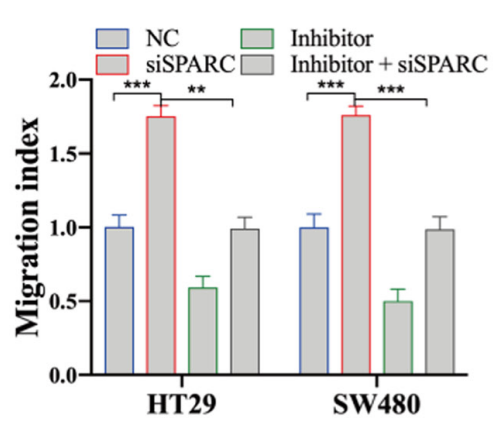

$24 \mathrm{~h}$
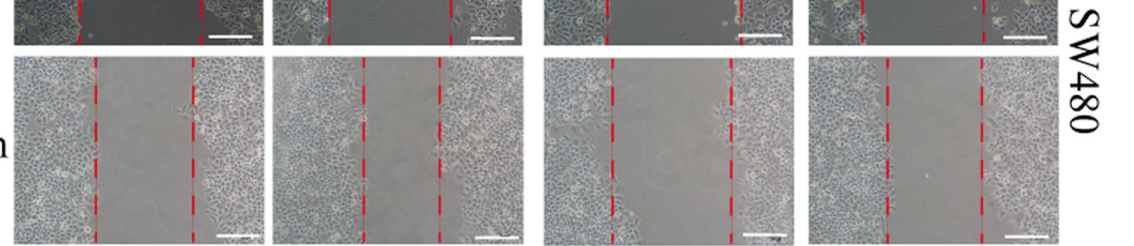

E

$\mathrm{NC}$

SiSPARC

Inhibitor Inhibitor + siSPARC
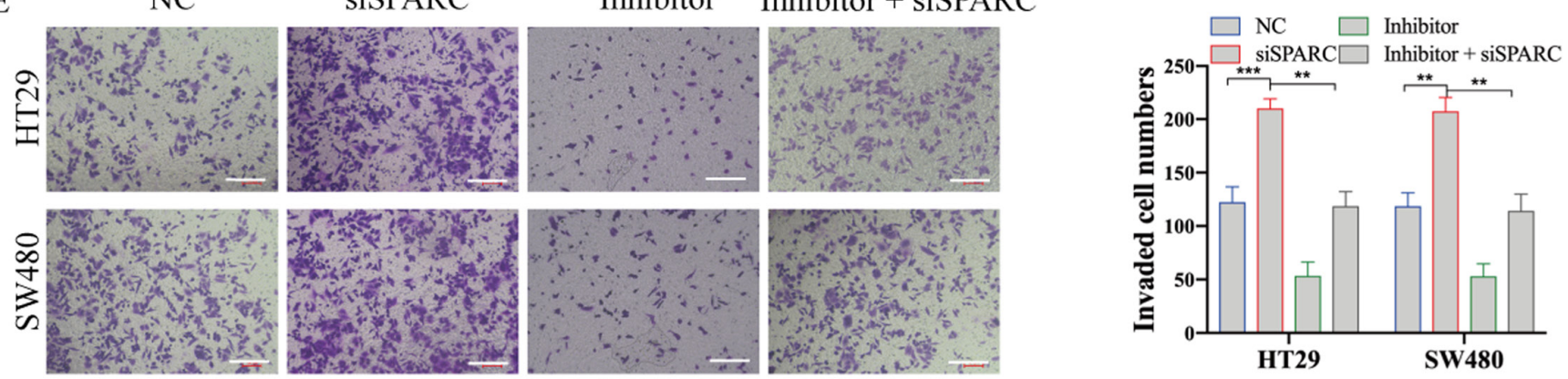

Figure 5. Knockdown of SPARC significantly reverses the effect of miR-592 knockdown on CRC cells. (A) Transfection effect of siSPARC and miR-592 inhibitor on SPARC expression in CRC cells. (B) Cell Counting Kit-8 and (C) incorporation assays demonstrated that siSPARC significantly promoted CRC cell proliferation, but co-transfecting with the miR-592 inhibitor reversed such effects.(D) Wound healing assay results indicated that siSPARC significantly promoted CRC cell migration, but co-transfecting with miR-592 inhibitor reversed these effects. (E) Transwell invasion assay results identified that siSPARC significantly promoted CRC cell invasion, but co-transfecting with miR-592 inhibitor reversed such effects. Scale bar, $100 \mu \mathrm{m} .{ }^{* * *} \mathrm{P}<0.01,{ }^{* * * *} \mathrm{P}<0.001$. NC, negative control; CRC, colorectal cancer; si, small interfering RNA; miR, microRNA; OD, optical density; SPARC, secreted protein acidic and rich in cysteine; EdU, 5-Ethynyl-2'-deoxyuridine 


\section{Availability of data and materials}

The datasets used and/or analyzed during the current study are available from the corresponding author on reasonable request. The GEO datasets analyzed during the current study are available in the GEO repository, (https://www.ncbi.nlm. nih.gov/geo/query/acc.cgi?acc=GSE128446; https://www. ncbi.nlm.nih.gov/geo/query/acc.cgi?acc=GSE126093; https://www. ncbi.nlm.nih.gov/geo/query/acc.cgi?acc=GSE125961).

\section{Authors' contributions}

CG and ZP designed the present study and drafted the initial manuscript. RX and WS acquired the clinical samples and performed RT-qPCR. Both RX and ZP performed the statistical analysis. CG and WS assessed the authenticity of all the raw data. All authors read and approved the final manuscript.

\section{Ethics approval and consent to participate}

The present study was approved by the Research and Ethical Committee at The Affiliated Huai'an No. 1 People's Hospital of Nanjing Medical University, and written informed consent was provided by all patients before the start of the study.

\section{Patient consent for publication}

Not applicable.

\section{Competing interests}

The authors declare that they have no competing interests.

\section{References}

1. Brody H: Colorectal cancer. Nature 521: S1, 2015.

2. Ferlay J, Soerjomataram I, Dikshit R, Eser S, Mathers C, Rebelo M, Parkin DM, Forman D and Bray F: Cancer incidence and mortality worldwide: Sources, methods and major patterns in GLOBOCAN 2012. Int J Cancer 136: E359-E386, 2015.

3. Mai D, Ding P, Tan L, Zhang J, Pan Z, Bai R, Li C, Li M, Zhou Y, Tan W, et al: PIWI-interacting RNA-54265 is oncogenic and a potential therapeutic target in colorectal adenocarcinoma. Theranostics 8: 5213-5230, 2018.

4. Dalmay T: Mechanism of miRNA-mediated repression of mRNA translation. Essays Biochem 54: 29-38, 2013.

5. Zhao H, Zheng C, Wang Y, Hou K, Yang X, Cheng Y, Che X, Xie S, Wang S, Zhang T, et al: miR-1323 promotes cell migration in lung adenocarcinoma by targeting Cbl-b and is an early prognostic biomarker. Front Oncol 10: 181, 2020.

6. Molist C, Navarro N, Giralt I, Zarzosa P, Gallo-Oller G, Pons G, Magdaleno A, Moreno L, Guillén G, Hladun R, et al: miRNA-7 and miRNA-324-5p regulate alpha9-Integrin expression and exert anti-oncogenic effects in rhabdomyosarcoma. Cancer Lett 477: 49-59, 2020.

7. Gharib E, Nasri Nasrabadi P and Reza Zali M: miR-497-5p mediates starvation-induced death in colon cancer cells by targeting acyl-CoA synthetase-5 and modulation of lipid metabolism. J Cell Physiol 235: 5570-5589, 2020.

8. Liu X, He B, Xu T, Pan Y, Hu X, Chen X and Wang S: MiR-490-3p functions as a tumor suppressor by inhibiting oncogene VDAC1 expression in colorectal cancer. J Cancer 9: 1218-1230, 2018.

9. Hou W, Zhang H, Bai X, Liu X, Yu Y, Song L and Du Y: Suppressive role of miR-592 in breast cancer by repressing TGF- $\beta 2$. Onco Rep 38: 3447-3454, 2017.
10. Li Z, Li B, Niu L and Ge L: miR-592 functions as a tumor suppressor in human non-small cell lung cancer by targeting SOX9. Oncol Rep 37: 297-304, 2017.

11. Gao S, Chen J, Wang Y, Zhong Y, Dai Q, Wang Q and Tu J: MiR-592 suppresses the development of glioma by regulating Rho-associated protein kinase. Neuroreport 29: 1391-1399, 2018.

12. He Y, Ge Y, Jiang M, Zhou J, Luo D, Fan H, Shi L, Lin L and Yang L: MiR-592 promotes gastric cancer proliferation, migration, and invasion through the PI3K/AKT and MAPK/ERK signaling pathways by targeting Spry2. Cell Physiol Biochem 47: 1465-1481, 2018.

13. Fu Q, Du Y, Yang C, Zhang D, Zhang N, Liu X, Cho WC and Yang Y: An oncogenic role of miR-592 in tumorigenesis of human colorectal cancer by targeting Forkhead Box O3A (FoxO3A). Expert Opin Ther Targets 20: 771-782, 2016.

14. Liu Z, Wu R, Li G, Sun P, Xu Q and Liu Z: MiR-592 inhibited cell proliferation of human colorectal cancer cells by suppressing of CCND3 expression. Int J Clin Exp Med 8: 3490-3497, 2015.

15. Cheng L, Xing Z, Zhang P and Xu W: Long non-coding RNA LINC00662 promotes proliferation and migration of breast cancer cells via regulating the miR-497-5p/EglN2 axis. Acta Biochim Pol 67: 229-237, 2020

16. Chen Y, Wu N, Liu L, Dong H and Liu X: microRNA-128-3p overexpression inhibits breast cancer stem cell characteristics through suppression of Wnt signalling pathway by down-regulating NEK2. J Cell Mol Med 24: 7353-7369, 2020.

17. Livak KJ and Schmittgen TD: Analysis of relative gene expression data using real-time quantitative PCR and the 2(-Delta Delta C(T)) method. Methods 25: 402-408, 2001.

18. Chen Z, Ren R, Wan D, Wang Y, Xue X, Jiang M, Shen J, Han Y, Liu F, Shi J, et al: Hsa_circ_101555 functions as a competing endogenous RNA of miR-597-5p to promote colorectal cancer progression. Oncogene 38: 6017-6034, 2019.

19. Kanehisa M and Goto S: KEGG: Kyoto encyclopedia of genes and genomes. Nucleic Acids Res 28: 27-30, 2000.

20. Colwill K and Gräslund S; Renewable Protein Binder Working Group: A roadmap to generate renewable protein binders to the human proteome. Nat Methods 8: 551-558, 2011.

21. Slattery ML, Herrick JS, Mullany LE, Samowitz WS, Sevens JR, Sakoda L and Wolff RK: The co-regulatory networks of tumor suppressor genes, oncogenes, and miRNAs in colorectal cancer. Genes Chromosomes Cancer 56: 769-787, 2017.

22. Wang W, Zhang H, Tang M, Liu L, Zhou Z, Zhang S and Wang L: MicroRNA-592 targets IGF-1R to suppress cellular proliferation, migration and invasion in hepatocellular carcinoma. Oncol Lett 13: 3522-3528, 2017.

23. Jia YY, Zhao JY, Li BL, Gao K, Song Y, Liu MY, Yang XJ, Xue Y, Wen AD and Shi L: miR-592/WSB1/HIF-1 $\alpha$ axis inhibits glycolytic metabolism to decrease hepatocellular carcinoma growth. Oncotarget 7: 35257-35269, 2016.

24. Lv Z, Rao P and Li W: MiR-592 represses FOXO3 expression and promotes the proliferation of prostate cancer cells. Int J Clin Exp Med 8: 15246-15253, 2015.

25. Liu M, Zhi Q, Wang W, Zhang Q, Fang T and Ma Q: Up-regulation of miR-592 correlates with tumor progression and poor prognosis in patients with colorectal cancer. Biomed Pharmacother 69: 214-220, 2015.

26. Cheetham S, Tang MJ, Mesak F, Kennecke H, Owen D and Tai IT: SPARC promoter hypermethylation in colorectal cancers can be reversed by 5-Aza-2'deoxycytidine to increase SPARC expression and improve therapy response. Br J Cancer 98: 1810-1819, 2008.

27. Drev D, Harpain F, Beer A, Stift A, Gruber ES, Klimpfinger M, Thalhammer S, Reti A, Kenner L, Bergmann M, et al: Impact of fibroblast-derived SPARC on invasiveness of colorectal cancer cells. Cancers (Basel) 11: 11, 2019.

28. Deng Z, Wu J, Xu S, Chen F, Zhang Z, Jin A and Wang J: Exosomes-microRNAs interacted with gastric cancer and its microenvironment: A mini literature review. Biomarkers Med 14: 141-150, 2020.

29. Kim SM, Yang Y,Oh SJ,Hong Y, Seo M and Jang M: Cancer-derived exosomes as a delivery platform of CRISPR/Cas9 confer cancer cell tropism-dependent targeting. J Control Release 266: 8-16, 2017.

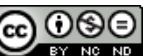

This work is licensed under a Creative Commons Attribution-NonCommercial-NoDerivatives 4.0 International (CC BY-NC-ND 4.0) License. 